\title{
Uma Causa Rara de Angina Após Revascularização do Miocárdio: Fístula da Artéria Mamária Interna Esquerda para Artéria Pulmonar e Tratamento Bem-Sucedido com Embolização Transcateter com Molas
}

\author{
A Rare Cause of Angina After Coronary Bypass Grafting; Left Internal Mammary Artery to Pulmonary Artery \\ Fistula and Successful Treatment with Transcatheter Coil Embolization
}

Ali Nazmi Calik, ${ }^{10}$ Can Yücel Karabay, ${ }^{1}$ Evliya Akdeniz, ${ }^{1}$ Yiğit Çanga, ${ }^{1}$ Baris Gungor, ${ }^{1}$ Omer Kozan ${ }^{1}$

Doktor Siyami Ersek Gogus Kalp ve Damar Cerrahisi Egitim ve Arastirma Hastanesi, ${ }^{1}$ Istanbul - Turquia

\section{Resumo}

A fístula da artéria mamária interna esquerda (AMIE) para a artéria pulmonar (AP) é raramente encontrada na prática diária. Nos últimos anos, opções de terapia endovascular surgiram para o tratamento de formações de fístula e foram substituídas por cirurgia.

Um homem de 53 anos de idade, internado em nosso ambulatório com sintomas de angina típica e falta de ar, apesar da terapia clínica ideal. Em seu histórico relevante, ele teve uma cirurgia de revascularização miocárdica (CRM) em 2009, na qual sua AMIE foi anastomosada à descendente anterior esquerda (DAE) e à artéria ramus sequencialmente. A angiografia coronária, incluindo imagens seletivas da AMIE, demonstrou uma formação de fístula proveniente da porção proximal da AMIE e drenando para AP. Após o fechamento bem-sucedido da fístula com embolização transcateter com mola, o paciente recebeu alta sem qualquer complicação e sintoma.

Em conclusão, embora fístula entre AMIE e AP seja uma condição clínica pouco frequente, deve ser considerada como uma causa potencial de angina persistente após a operação de revascularização do miocárdio. As opções de tratamento incluem terapia médica conservadora, ligadura cirúrgica e intervenções endovasculares. A melhor terapia deve ser individualizada para cada paciente em relação aos sintomas do paciente, compatibilidade cirúrgica e anatomia da fístula.

\section{Introdução}

A artéria mamária interna esquerda (AMIE) é o vaso mais comumente usado como um canal para o enxerto de bypass para a artéria descendente anterior esquerda (DAE) devido à sua longa patência. Embora a fístula entre AMIE e artéria pulmonar (AP) seja uma condição clínica rara, a cirurgia de

\section{Palavras-chave}

Artéria Torácica Interna/cirurgia; Artéria Pulmonar; Fístula Artério-Arterial; Embolização Terapêutica; Cateterismo Cardíaco; Stents Farmacológicos; Stents Metálicos Autoexpansíveis.

Correspondência: Ali Nazmi Calik •

Doktor Siyami Ersek Gogus Kalp ve Damar Cerrahisi Egitim ve Arastirma

Hastanesi - Cardiology, 34664, Istanbul - Turquia

E-mail: calik_nazmi@hotmail.com

Artigo recebido em 03/07/19, revisado em 14/11/2019, aceito em 14/11/2018

DOI: $10.5935 / a b c .20190196$ revascularização do miocárdio (CRM) é uma causa comum das fístulas de AMIE para AP adquiridas. Essa situação clínica é importante em relação à angina recorrente, apesar da terapia clínica ideal após a CRM. Atualmente, as intervenções endovasculares têm sido aplicadas com sucesso no tratamento das formações de fístulas e são consideradas como opções terapêuticas de primeira linha. Neste trabalho, relatamos uma fístula AMIE para AP e tratamento bem-sucedido com embolização transcateter com mola.

\section{Relato de caso}

Paciente do sexo masculino, 53 anos, com histórico de revascularização miocárdica em dois vasos, em 2009, em que sua AMIE foi anastomosada à DAE e à artéria ramus, internado sequencialmente em nosso ambulatório com sintomas de angina típica e falta de ar, apesar da terapia clínica ideal. A angiografia coronária demonstrou estenose de $60 \%$ da artéria coronária direita (CD) proximal, com um valor de FFR de 0,87 , $90 \%$ de estenose da artéria ramus nativa e formação de fístula de AMIE para AP antes de anastomosar para DAE e artéria ramus (Figura 1). A angiotomografia computadorizada (TC) de artérias coronárias e revascularização do miocárdio confirmou o diagnóstico de fístula e seu trajeto. Depois de consultar o paciente com a equipe do coração, incluindo cirurgiões cardiotorácicos e anestesiologistas no sentido de estratégia de tratamento, decidimos revascularizar completamente o paciente com stent da artéria ramus nativa e fechar a fístula com embolização por embolização transcateter com mola no mesmo procedimento. Nossa justificativa para o tratamento da artéria ramus nativa no mesmo procedimento visava bloquear o fluxo sequencial proveniente da AMIE competindo com a mesma, o que resultaria no aumento do fluxo de AMIE para DAE.

Após implante de stent na artéria ramus com stent farmacológico de 3,0 × $15 \mathrm{~mm}$, acoplamos cuidadosamente o óstio da AMIE com cateter guia número 8 às artérias mamárias internas (AMI) e avançamos um guia de 0,014 " para a parte distal da fístula. Em seguida, um microcateter CANTATA francês 2,8 (Cook Medical, Bloomington, IN, EUA) foi avançado para a porção média da fístula sobre o guia PT2. Como a fístula estava drenando para AP com dois ramos laterais principais que se dividiam em múltiplos colaterais em suas porções distais, nossa estratégia inicial era ocluir os dois principais ramos da fístula individualmente. No entanto, a primeira mola (espiral de 11-2-2 HL, Sistema de mola de Detach, Cook Medical, Bloomington, IN, EUA) foi embolizada distalmente inadvertidamente e as outras duas bobinas (espiral de 11-3-4 HL 


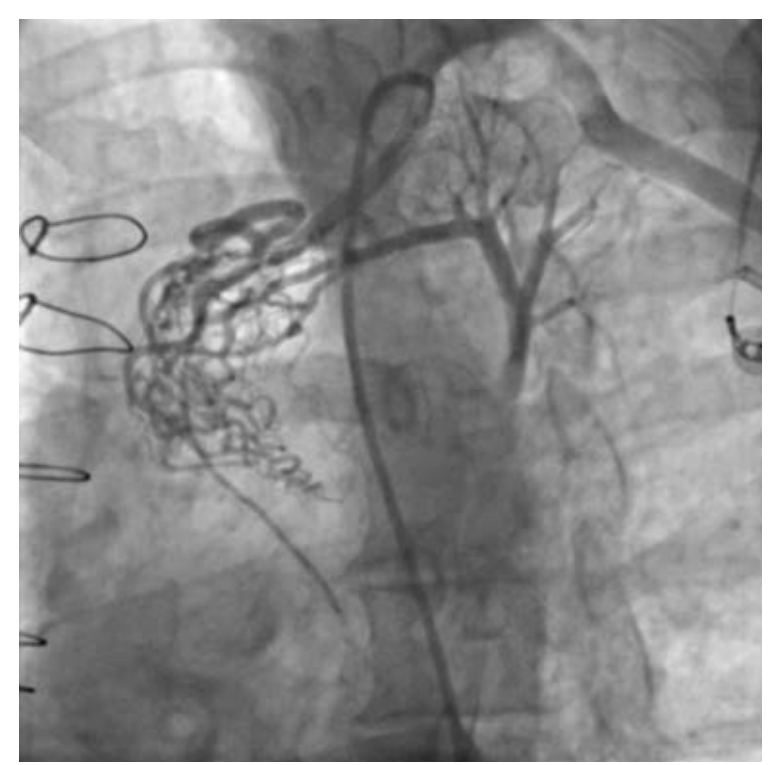

Figura 1 - Visão angiográfica da formação de fístula entre LIMA e artéria pulmonar

e espiral de 18-4-6 HL) para cada ramo lateral não pôde ocluir completamente a fístula (Figura 2). Assim, mudamos nossa estratégia e implantamos mais três molas maiores (espiral 18S-5-7 HL, espiral 18S-6-8 HL, espiral 18S-6-15 HL) no ramo principal da fístula. Após aguardar alguns minutos, a angiografia de controle da AMIE demonstrou oclusão total da fístula sem nenhuma complicação (Figura 3). O paciente tolerou bem o procedimento e recebeu alta sem complicações e sintomas.

\section{Discussão}

A formação de fístula da AMIE para AP é uma entidade clínica incomum. O primeiro caso de fístula da AMIE para AP foi apresentado por Burchell e Clagett ${ }^{1}$ em 1947. Desde então, tais casos foram publicados muito raramente. As fístulas da AMIE para a AP podem ser congênitas ou adquiridas. A causa comum da forma adquirida é a CRM, mas também pode ser secundária a trauma, infecção e neoplasia. ${ }^{2-4}$ Madu et al., ${ }^{5}$ relatou a incidência de fístula da AMIE para de AP como 0,67\% por meio da revisão de 595 angiogramas pós-revascularização miocárdica e a média para o desenvolvimento de tal fístula foi de cerca de 5 anos. Um estudo semelhante, conduzido por Guler et al., ${ }^{6}$ revisou 537 angiogramas coronários de pacientes pós-revascularização do miocárdio e relatou que apenas 5 $(0,93 \%)$ deles tinham formação de fístula com origem na AMIE e drenagem para vasculatura pulmonar.

Vários mecanismos fisiopatológicos têm sido propostos para a formação de fístula da AMIE para a AP, embora a causa subjacente não seja totalmente compreendida. Entretanto, fatores predisponentes para o desenvolvimento da fístula incluem: o uso de eletrocautério em vez de ligadura dos ramos laterais durante a retirada da $\mathrm{AMIE},{ }^{7,8}$ lesão da pleura e do parênquima pulmonar ${ }^{9}$ que pode causar contato direto entre AMIE e a vasculatura pulmonar, infecção do local de operação e processo inflamatório levando à neovascularização.
O sintoma mais comumente relatado é angina persistente, apesar da terapia clínica ideal. Fenômeno de fuga de fluxo coronário e isquemia miocárdica devido a desvio substancial de sangue da AMIE para a AP é proposto como o mecanismo fisiopatológico subjacente da angina. ${ }^{5}$ No entanto, os pacientes podem consultar os médicos com dispnéia e outros sintomas da insuficiência cardíaca congestiva. O sopro contínuo pode ser ouvido no exame físico. Embora seja muito incomum, tal formação de fístula pode ser complicada por infarto do miocárdio, insuficiência cardíaca congestiva, hipertensão pulmonar, arterite, aneurisma ou ruptura. ${ }^{6}$

A angiografia seletiva da AMIE é de extrema importância para diagnosticar essa formação de fístula. ${ }^{10}$ As opções de tratamento da fístula AMIE-AP incluem terapia medicamentosa conservadora, ligadura cirúrgica e opções de terapia endovascular, como embolização com mola ou plugue vascular e implante de stent revestido. Pacientes assintomáticos com pequenas fístulas e pacientes cirúrgicos de alto risco que não têm anatomia adequada para intervenções endovasculares podem ser acompanhados por terapia médica conservadora; no entanto, pacientes que sofrem de angina e sintomas de insuficiência cardíaca congestiva, apesar da terapia clínica ideal, e pacientes com dilatação aneurismática da fístula devem ser intervencionados cirurgicamente ou percutaneamente. Atualmente, as opções terapêuticas endovasculares menos invasivas substituem o tratamento cirúrgico, o que acarreta um risco significativo de morbidade e mortalidade.

As técnicas de embolização com mola e plugue vasculares são as opções de terapia endovascular mais utilizadas para o tratamento de formações de fístula. No entanto, ambas as técnicas têm prós e contras. Embolização com mola é geralmente usada para fístulas menores ${ }^{11}$ e precisa de cateteres menores para a colocação. Além disso, as molas são dispositivos discretos, fáceis de colocar e de 


\section{Relato de Caso}

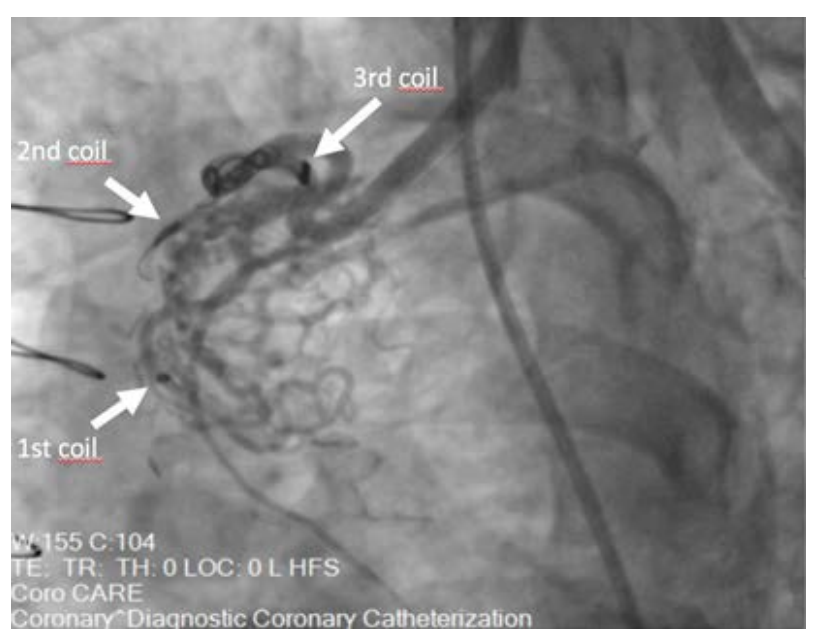

Figura 2 - Visão angiográfica após as três primeiras bobinas que não conseguiram obstruir completamente a fistula.

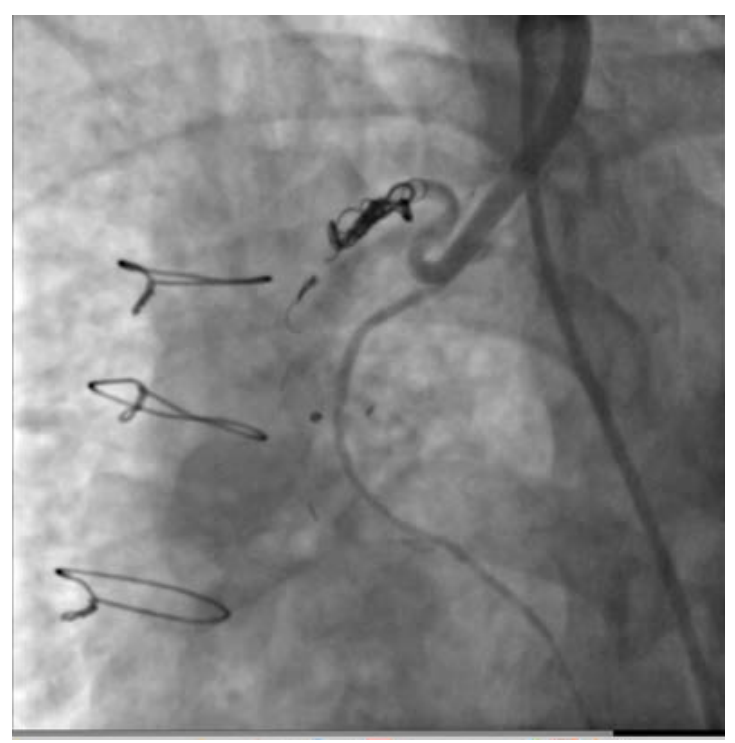

Figura 3 - Oclusão total da fístula após embolização adicional da bobina.

baixo custo quando comparadas aos plugues vasculares. Mas, o calcanhar de Aquiles da embolização com mola são altas taxas de recanalização e embolização distal, como tivemos em nosso caso. Por outro lado, a embolização com plugue vascular é geralmente preferida para fístulas grandes ${ }^{11}$ e permite um posicionamento mais preciso com menos taxas de embolização e recanalização distal. No entanto, os plugues vasculares são mais caros que as molas e requerem cateteres maiores para a colocação (número 4, no mínimo). Além disso, o posicionamento e a implantação em vasos tortuosos podem ser desafiadores em alguns casos devido ao conjunto rígido de fios de liberação do cateter. ${ }^{12}$ Além das características individuais desses dispositivos, a decisão do tipo de terapia endovascular depende da experiência do operador e da anatomia da fístula.

Dito isto, uma vez que o nosso caso sofria de angina persistente apesar da terapia clínica ideal e refazer a operação constituía um risco cirúrgico significativo, decidimos tratar o paciente com intervenção endovascular. Além disso, a formação de fístula foi adequada para embolização transcateter com mola em termos de diâmetro e anatomia. Como a fístula apresentava uma estrutura tortuosa, particularmente em sua porção proximal, não utilizamos oclusor de plugue vascular neste caso. 


\section{Conclusão}

As fístulas AMIE-AP são condições raras que podem se desenvolver após a revascularização do miocárdio e devem ser lembradas como causa de angina persistente. A angiografia seletiva da AMIE com avaliação cuidadosa das imagens é substancial para o diagnóstico e tratamento adequado desta entidade. As intervenções endovasculares são agora consideradas opções terapêuticas de primeira linha. A experiência médica e institucional, bem como a anatomia e as características da fístula, são cruciais ao decidir o tipo de terapia endovascular.

\section{Contribuição dos autores}

Concepção e desenho da pesquisa: Calik AN, Karabay CY, Gungor B, Kozan O; Obtenção de dados: Calik AN, Karabay $\mathrm{CY}$, Akdeniz E, Çanga Y, Kozan O; Redação do manuscrito: Calik AN, Akdeniz E, Çanga Y, Gungor B, Kozan O.

\section{Potencial conflito de interesses}

Declaro não haver conflito de interesses pertinentes.

\section{Fontes de financiamento}

O presente estudo não teve fontes de financiamento externas.

\section{Vinculação acadêmica}

Não há vinculação deste estudo a programas de pós-graduação.

\section{Aprovação ética e consentimento informado}

Este artigo nao contem estudos com humanos ou animais realizados por nenhum dos autores.

\section{Referências}

1. Burchell HB, Clagett OT. The clinical syndrome associated with pulmonary arteriovenous fistulas, including a case report of a surgical cure. Am Heart J $1947 ; 34(2): 151-62$

2. Pierce G, Ahuja C, Chadha M. Case report: complex internal mammary to pulmonary artery fistula as a cause of hemoptysis in tuberculosis: diagnosis and endovascular management using ethylene vinyl alcohol copolymer (Onyx). Indian J Radiol Imaging. 2011;21(1):10-2.

3. Ito T, Sakamoto T, Norio H, Kaji T, Okada Y. An arteriovenous fistula between the internal mammary artery and the pulmonary vein following blunt chest trauma. Cardiovasc Intervent Radiol. 2005;28(1):120-3.

4. DunnRP, Wexler L. Systemic-to-pulmonary fistula in intrapulmonary Hodgkin's disease. Chest. 1974;66(5):590-4.

5. Madu EC, Hanumanthu SK, Kim C, Prudoff A. Recurrent ischemia resulting from left internal mammary artery-to-pulmonary artery fistula. Angiology. 2001;52(3):185-8

6. Guler A, Yildiz M, Karabay CY, Aung SM, Aykan AC, Karagoz A, et al. Case series of a rare complication of CABG Fistula between the internal

mammary artery and pulmonary vasculature. Herz. 2014;39(1): 149-53.

7. Wood MK. Internal mammary artery to lung parenchyma fistula. Ann Thorac Surg. 1992;54(3):603.

8. Blanche C. Internal mammary artery to lung parenchyma fistula. Reply (Letter). Ann Thorac Surg. 1992;54(3):603.

9. Groh WJ, Hovaguimian $\mathrm{H}$, Morton MJ. Bilateral internal mammary to pulmonary artery fistulas after coronary operation. Ann Thorac Surg. 1994; 57(6):1642-3.

10. Peter AA, Ferreira AC, Zelnick K, Sangosanya A, Chirinos J, de Marchena E. Internal mammary artery to pulmonary vasculature fistula case series. Int J Cardiol. 2006;108(1):135-8.

11. Girona J, Martí G, Betrián P, Gran F, Casaldàliga J. Percutaneous embolization of vascular fistulas using coils or Amplatzer vascular plugs. Rev Esp Cardiol. 2009;62(7):765-73

12. Lopera JE. The Amplatzer Vascular Plug: Review of Evolution and Current Applications. Semin Intervent Radiol. 2015;32(4):356-69. 